\title{
Enhancing Continuous Control of Mobile Robots for End-to-End Visual Active Tracking
}

\author{
Alessandro Devo, Alberto Dionigi, Gabriele Costante
}

\begin{abstract}
In the last decades, visual target tracking has been one of the primary research interests of the Robotics research community. The recent advances in Deep Learning technologies have made the exploitation of visual tracking approaches effective and possible in a wide variety of applications, ranging from automotive to surveillance and human assistance. However, the majority of the existing works focus exclusively on passive visual tracking, i.e., tracking elements in sequences of images by assuming that no actions can be taken to adapt the camera position to the motion of the tracked entity. On the contrary, in this work, we address visual active tracking, in which the tracker has to actively search for and track a specified target. Current State-of-the-Art approaches use Deep Reinforcement Learning (DRL) techniques to address the problem in an endto-end manner. However, two main problems arise: i) most of the contributions focus only on discrete action spaces and the ones that consider continuous control do not achieve the same level of performance; and ii) if not properly tuned, DRL models can be challenging to train, resulting in a considerably slow learning progress and poor final performance. To address these challenges, we propose a novel DRL-based visual active tracking system that provides continuous action policies. To accelerate training and improve the overall performance, we introduce additional objective functions and a Heuristic Trajectory Generator (HTG) to facilitate learning. Through an extensive experimentation, we show that our method can reach and surpass other State-of-the-Art approaches performances, and demonstrate that, even if trained exclusively in simulation, it can successfully perform visual active tracking even in real scenarios.
\end{abstract}

\section{INTRODUCTION}

The capability to detect and track a target object across multiple frames collected by vision sensors, i.e. Visual Tracking (VT), plays an important role in many Robotic researches. The possible applications of VT technologies span across different areas, e.g., autonomous driving, surveillance, robot manipulation and human assistance, to name a few. In the last years, the advent of Deep Learning-based techniques has exponentially increased the progresses made in order to improve both performance and robustness of VT algorithms. In particular, the use of Convolutional Neural Networks (CNNs) has allowed the development of increasingly sophisticated and precise tracking systems. However, most of the works focus exclusively on tracking objects and/or people in pre-recorded videos [1], [2], [3], [4] or, in general, assume that the target is always within the field of view of a fixed camera, whose position cannot be adapted to the target

The authors are with the Department of Engineering, University of Perugia, 06125 Perugia, Italy alessandro.devodstudenti.unipg.it, alberto.dionigiestudenti.unipg.it,

gabriele.costante@unipg.it motion. This condition considerably limits the possibility to apply VT approaches in many real-world robotic scenarios.

Therefore, in this work, we focus on the more challenging task of Visual Active Tracking (VAT) [5], [6], [7], in which the tracker has to actively search for and track a specified target (Fig. 1). This task is clearly more complex than passive VT, since the tracker must not only identify the target but also act and change its position to maintain view contact with it. This requires the robot to localize itself with respect to the target and plan in real-time the most suitable trajectory to follow it.

A possible naive way to tackle the problem is to combine a generic object detection module, such as [8], with other localization, collision detection and planning techniques [9], [10], [11], [12], [13]. However, since this strategy is not specifically designed for target tracking, it is highly inefficient and generates a considerable information overhead.

A better solution is to get rid of the localization, mapping and collision detection blocks and focus only on two separate modules: the first has to identify and passively tracks the target in the images; the second is responsible for planning the tracker trajectory to follow the target [14], [15], [16]. Although this solution has brought interesting results, it still has some inefficiencies and the non-trivial problem of combining the two components remains.

For this reason, more recent works [6], [7] propose the use of Deep Reinforcement Learning (DRL) algorithms to address the problem in an end-to-end manner. DRL has proven to be remarkably effective in many vision, navigation and robotics tasks, particularly when multiple mutual objectives need to be accomplished simultaneously [17], [18], [19], [20], [21]. DRL-based VAT systems are able to process the image stream directly and provide action policies to adapt the camera field of view frame-by-frame, avoiding the need to manually adapt two separate vision and motion modules.

The well-known shortcomings of DRL algorithms, i.e., i) the need for a considerable amount of training samples for their optimization and ii) their trial-and-error learningbased nature have been addressed by relying upon synthetic environments [6], [7]. The availability of graphic engines with increased level of photorealism and the exploitation of recent sim-to-real domain adaptation techniques have made it possible to bridge the domain gap between DRL models trained on simulated scenarios and the real world without the need for fine-tuning procedures. In particular, among domain adaptation strategies, domain randomization [22] is certainly one of the most popular and has been successfully applied to various robotic tasks [23], [18] and even to 

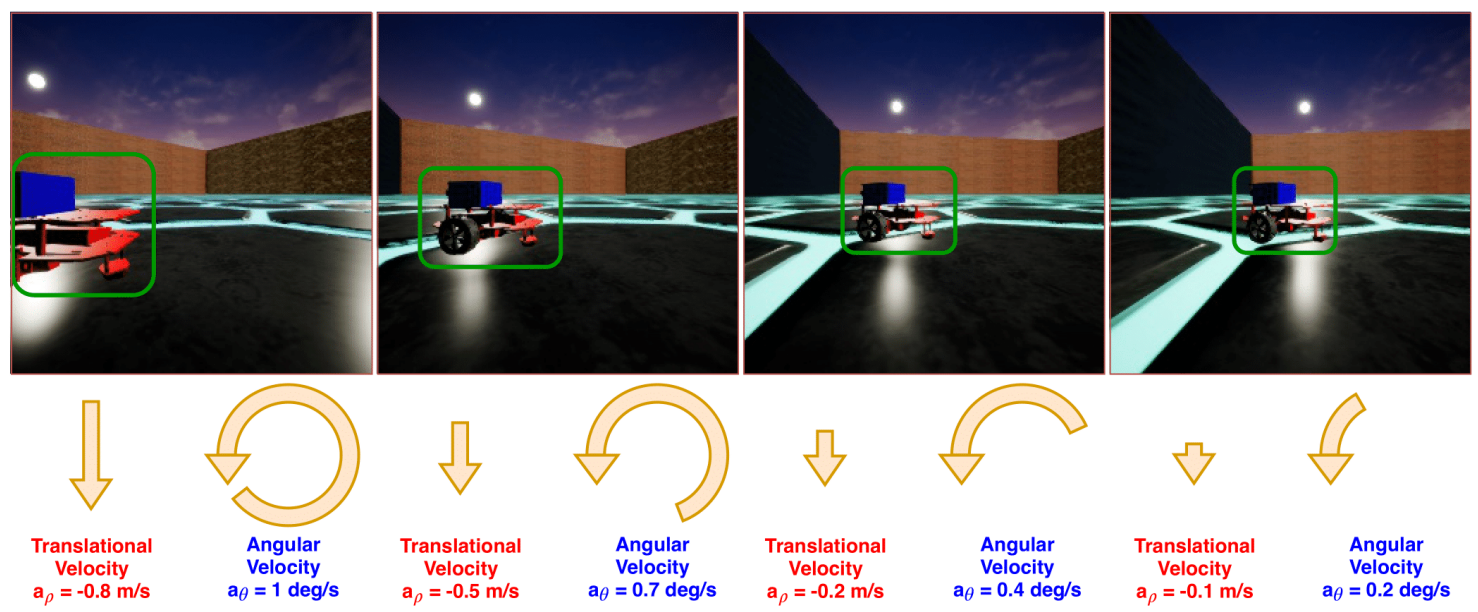

Fig. 1. The visual active tracking task. The goal of the tracker is to maintain the target (marked in green) within its field of view. Contrary to passive tracking, which assume that the target is always within the field of view of a fixed camera, in the active scenario the tracker has to perform motion maneuvers to maintain view contact with it. In this example (from left to right), the tracker chooses to consecutively perform backward translation and left turning to center the target.

VAT [7]. However, most of these DRL approaches for VAT only consider discrete action spaces. On the other hand, continuous control methods are often difficult and slow to train, resulting in optimization procedures that require tens of millions of steps before starting to learn [6] or may not even be successful.

For these reasons, in this work, we propose C-VAT, a novel DRL-based approach for VAT in continuous action spaces, which features a new training procedure that produces sample efficient, effective and robust visual tracking policies. Specifically, we employ a deterministic tracking algorithm to considerably speed up the critic component learning and an auxiliary loss that, since the beginning of training, helps the actor to develop a useful basic understanding of the tracking task. Our approach is trained exclusively in simulated environments and benefits from domain randomization to achieve generalization over real world contexts. We evaluate our method performance in a large variety of synthetic experiments, and show that, even if trained with synthetic data only, our algorithm can be effectively used in real scenarios with physical robots.

This work proceeds as follows: Section III contains our literature review; Section III formalizes the task, presents our approach and the environment setup; Section IV describes the experiments and shows the results; finally, Section V draws our conclusions and the path of future work.

\section{RELATED WORK}

The majority of the state-of-the-art works focus on passive visual tracking [1], [2], [3], [4] i.e., they assume that the camera cannot be moved to react to the target object motions and that the latter is always in the camera field of view. The authors in [24], for example, propose a novel distractor-aware approach based on Siamese networks for visual object tracking. Conversely, in [25], an adversarial training technique is introduced. They perform data augmentation by using a generative neural network to generate useful masks. The one that maintains the best features is then identified by the network through adversarial learning. Finally, the authors of [26] devise a novel DRL-based tracking approach that iteratively moves an initial bounding box to follow the target across the image sequence.

Despite the recent advances, the passive approach is still limited to cases in which the target is within the camera field of view. On the contrary, active tracking approaches cover a wider range of situations since they can take advantage of the mobility of the hardware in which they are installed, e.g., movable surveillance cameras or mobile robots. Various approaches [14], [15], [16] try to combine passive models with camera control modules to actively perform tracking. [27] introduces a real-time visual tracking system for indoor human motion tracking, which separates the task in image acquisition and camera motion estimation, object motion detection and localization, and camera control. In [28], a hierarchical system to control a set of surveillance cameras is presented. In [5], the authors propose a system composed by three main different components to perform mapping, detecting and tracking the object. [29] introduces a new modular architecture that incorporates a model for perception and another one for the control policy. The authors used the former to produce a semantic image segmentation from the perceived RGB frame, which is then used by the latter to perform the actions.

While addressing the task with modular systems is possible, employing such solutions has many drawbacks. In particular, a flaw in one of the components can propagate to the entire system, causing an overall failure. Furthermore, combining the separates modules of visual tracking and camera control can be considerably expensive.

For these reasons, recent works are focusing on end-toend solutions, in which a direct mapping between vision and motion is learned. To this end, DRL has shown to be particular effective in many visual-navigation [30], [19], [20], 
[21] and robotics tasks [31], [32]. [18] proposes a DRL algorithm able to learn complex dexterous in-hand manipulation policies. [33] show how an agent can be trained, from raw image pixels, to effectively explore complex unknown mazes and find a specific target. [34] proposes a system that, from raw image observations only, can directly control torques at the robots motors. In [35], a system trained exclusively in simulation for collision-free indoor flight in real environments is designed.

Inspired by these successes, some works introduce DRLbased systems for end-to-end VAT [36], [6], [7]. In particular, [36] introduces a novel Pose-Assisted Multi-Camera Collaboration System composed by three main modules: the posebased controller, the vision-based controller and a switcher that, in each step, chooses the best controller based on the visibility of the target. [6] proposes a discrete action space CNN-LSTM model to track a human-like target, using only raw RGB frames as input. They also introduce a specifically designed reward function and an environment augmentation technique based on domain randomization [22], [23], [37], [38], [18] to generalize in real world scenarios. In addition, the authors propose a continuous variant of their method, which, however, due to the extra complexity of managing continuous actions, demonstrate lower performances that its discrete counterpart. Since in that work the target policy is fixed throughout all the learning process, [7] introduces an asymmetric duelling training procedure, during which the target learn complex escape policies to avoid to be tracked. The authors demonstrate that such a mechanism allows the tracker to practice with a much more challenging target, making it more robust and faster to train.

\section{A. Contribution}

Current state-of-the-art VAT methods [6], [7] primarily focus on discrete action spaces, which limit their applicability do not allow them to achieve performances comparable to those of a continuous control approach. Therefore, we propose a novel training procedure based on the popular Deep Deterministic Policy Gradient (DDPG) algorithm [39], which can successfully and consistently perform tracking with continuous actions. Due to the complexity of the task and the continuous action space and the off-policy nature of the DDPG algorithm, other works that simply apply DRL to train continuous action policies obtain extremely poor results, as we demonstrate in our experimental section. On the contrary, our approach can effectively handle continuous action spaces, overcoming the limitations of previous stateof-the-art continuous control methods, and can produce robust tracking policies, surpassing also discrete action models performance.

To summarize, our contribution is three-fold:

1) We introduce C-VAT, a DRL-based architecture that computes policies in continuous action space for endto-end VAT;

2) We devise a training technique that leverages different strategies to ease and speed-up the optimization

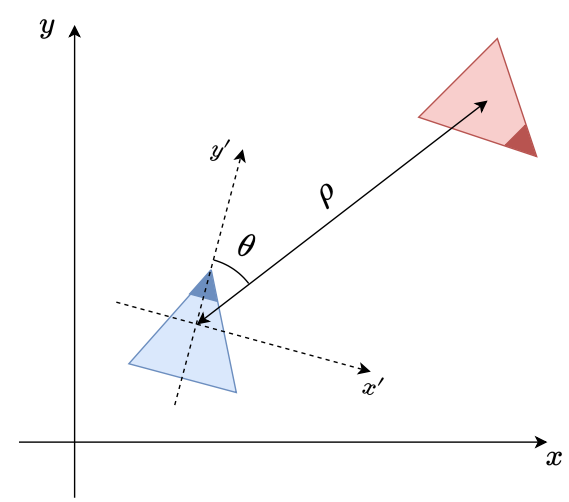

Fig. 2. The considered coordinate system to compute the reward $r$. As can be observed, $\rho$ represents the euclidean distance between the tracker (blue triangle) and the target (red triangle), and $\theta$ constitutes the angle defined by $\rho$ and the tracker forward direction. Both $\rho$ and $\theta$ are measured by considering the relative coordinate system w.r.t. the tracker.

process and achieve better performance than state-ofthe-art baselines;

3) We demonstrate that C-VAT, although trained exclusively on simulated environments, guarantees remarkable performance even in real scenarios, without the need for any tuning procedure.

\section{APPROACH}

In this section, we first formally define the problem and provide a concise background on the classical RL setting. Secondly, we describe how we can formulate our specific VAT task as a DRL problem. In the third section, we explain in detail the proposed training procedure and network architecture. Finally, we presents the simulated environments we use to train our model.

\section{A. Problem Formulation}

The objective of an autonomous robot that performs VAT, referred to in the following as tracker, is to recognize and actively track a predefined, and possibly moving, target, by using only visual inputs. Formally, we frame the task as a classic RL problem [40], in which an agent interacts with an environment $E$ over a discrete number of timesteps. The environment can be seen as a Markov Decision Process (MDP) in which the main task of the agent is to find a policy $\pi$ that maximizes the sum of discounted future reward:

$$
R_{t}=\sum_{i=t}^{T} \gamma^{i-t} r_{i}\left(x_{i}, a_{i}\right)
$$

where $\gamma \in[0,1)$ is the discount factor and $r_{i}\left(x_{i}, a_{i}\right)$ is the reward at time $i$, given the state $x_{i}$ and the action $a_{i} \sim$ $\pi\left(\cdot \mid x_{i}\right)$.

We can also define the action-value function $Q_{\pi}$ as:

$$
Q_{\pi}\left(x_{t}, a_{t}\right)=\mathbb{E}_{r_{i \geq t}, x_{i>t} \sim E, a_{i>t} \sim \pi}\left[R_{t} \mid x_{t}, a_{t}\right],
$$

which describes the expected return after choosing an action $a_{t}$ in state $x_{t}$ and thereafter following policy $\pi$. It should be 
noticed that estimating correctly $Q_{\pi}$ means knowing exactly which is the best action $a_{t}$ to take for every $x_{t}$, and hence, solving the MDP.

It is important to remark, however, that the true state $x_{t}$ of the environment can be unknown to the agent (as in our setting). In such a case, $E$ is, in fact, a Partially Observable MDP (POMDP), which, instead, provides to the agent only an observation $o_{t}$ of the underlying state.

In the following, we define what $x_{t}, o_{t}, a_{t}$ and $r_{t}$ represent in our particular scenario (see Section III-B) and describe the algorithm we employ to estimate $Q_{\pi}$ (see Section III-C).

\section{B. Task Details}

In our specific case, we consider a VAT system whose inputs consist of the RGB frame collected by the tracker camera (we assume it mounted in the front of the robot). Our tracker is free to move along the $\mathrm{X}$ and the $\mathrm{Y}$ axes of a three-dimensional space by performing continuous actions, in order to adapt its position to keep the target in its field of view. In this setting, we can notice that: i) a captured image represents only an observation $o_{t}$ of the unknown underlying state $x_{t}$ (i.e., the positions of the tracker and the target, and the map of the environment); ii) the observation space is extremely vast, since generated by all the possible combinations of pixel values; iii) the tracker action space is infinite, since we consider continuous controls. For these reasons, classical RL algorithms cannot be applied and the use of more advanced DRL methods that exploit complex Deep Neural Network (DNN) approximators is necessary.

We start by considering a series of independent episodes during which our agent (i.e., the tracker) interacts with the environment to collect visual observations, perform continuous control actions and get rewards. When an episode starts, the tracker has to first look around to find the target, since it can also spawn outside its initial field of view. Then, it can start to track it and maximize the reward signal $r_{t}$, which we defined as:

$$
r_{t}=A r_{\rho_{t}} r_{\theta_{t}}
$$

where

$$
r_{\rho_{t}}=\max \left(0,1-\frac{\left|\rho_{t}-\rho^{*}\right|}{\rho_{\max }}\right)
$$

and

$$
r_{\theta_{t}}=\max \left(0,1-\frac{\left|\theta_{t}-\theta^{*}\right|}{\theta_{\max }}\right) .
$$

In particular, in Eq. (4) $\rho_{t}$ represents the current distance between the tracker and the target while $\rho^{*}$ and $\rho_{\max }$ the optimal and the maximum ones, respectively. Consequently, $r_{\rho_{t}}$ encodes how close the distance between the tracker and the target is to the optimal one. Conversely, in Eq. (5), $r_{\theta_{t}}$ measures the tracker ability to maintain the target within its field of view, with $\theta_{t}, \theta^{*}$ and $\theta_{\max }$ indicating the current, desired and maximum angular distances, respectively. All the angles are computed with respect to the center of the field of view, hence, $\theta^{*}$ is set to $0^{\circ}$ if the tracker is required to keep the target in the center of the image (see Fig. 2).
From the equations, it can be noticed that $r_{t}$ is always included in the range $[0, A]$ (where $A$ is an algorithm hyperparameter) and is maximum when both $\rho_{t}$ and $\theta_{t}$ coincide exactly with $\rho^{*}$ and $\theta^{*}$, respectively. On the other end, $r_{t}$ is 0 if $\rho_{t}$ exceeds $\rho_{\max }$ or $\theta_{t}$ is greater than $\theta_{\max }$.

The tracker action space is continuous. At each timestep $t$, it produces two different and independent real valued actions, $a_{\rho_{t}}$ and $a_{\theta_{t}}$, which represents the translational and the angular speeds, respectively. It should be observed that such continuous actions allow extremely fine maneuvers that would not be possible with discrete ones.

Regardless of the reward received and the actions performed, a training episode ends when a predefined number of steps is reached.

During training, the target position is kept fixed, hence, the tracker job is simply to move itself in order to meet the desired $\theta^{*}$ and $\rho^{*}$ and, then, to maintain its current position and orientation. One could argue that, compared with other much more complex procedures (as those in used in [7]), training with such a simple task could prevent the tracker to generalize over more complex and general tracking scenarios, i.e., with moving targets. Nonetheless, in Section IV] we show that this simple training strategy is sufficient to learn robust tracking abilities for far more challenging scenarios.

\section{Network Architecture and Training Algorithm}

To train our model we use the Asynchronous Advantage Actor Critic (A3C) [41] framework combined with the DDPG algorithm [39] (see Algorithm 1), which is specifically designed to deal with continuous action spaces and DNN approximators.

We use several instances of our agent to collect trajectories of states, actions and rewards. All the copies have a local network, whose architecture is identical for all of them, which is periodically synchronised with a shared model. Each copy is placed in a different room, where it collects data and stores them in a personal replay buffer [42]. Every time an agent has to be updated, a number of samples are randomly picked from its buffer. The agent uses these data to compute the losses (specific details on Sections III-C.2 and III-C.4 and the gradients with respect to its local network. The gradients are then transferred to the shared network, which updates the parameters and sends them back to the local network. It should be noticed that since the agents are updated independently, the learning process is asynchronous and, due to the replay buffer, it is also off-policy.

In the following sections, we explain in major detail the various elements that compose the proposed approach.

1) Architecture Details: Similarly to other actor-critic algorithms, DDPG makes use of two main components: an actor, which chooses the actions to be performed, and a critic, which evaluates such actions. We implement these entities with two distinct DNNs, namely A-DNN for the actor and C-DNN for the critic, as shown in Fig. 3

A-DNN is fed with a $84 \times 84$ RGB frame $\left(o_{t}\right)$ that is initially processed by 2 convolutional layers: the first with 16 


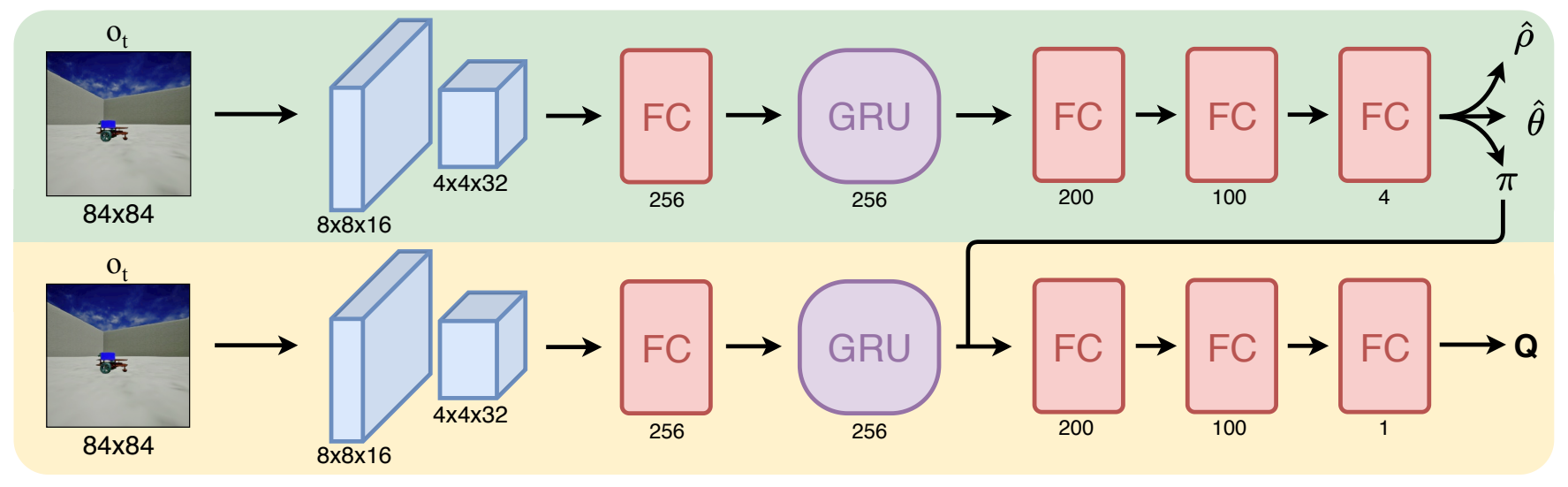

Fig. 3. The proposed architecture for visual active tracking. It is composed by two main components: the actor network (in green) and the critic network (in yellow). Both are fed with the current $84 \times 84 \mathrm{RGB}$ frame, which is first processed by the actor to produce the estimated relative distance $(\hat{\rho})$ and angle $(\hat{\theta})$ for the auxiliary angle-distance loss, and the policy vector $(\pi)$. The latter is then concatenated with the features extracted by the GRU of the critic, which, finally, outputs the Q-value function $(Q)$. Despite the two networks are almost identical, their weights are not shared between them.

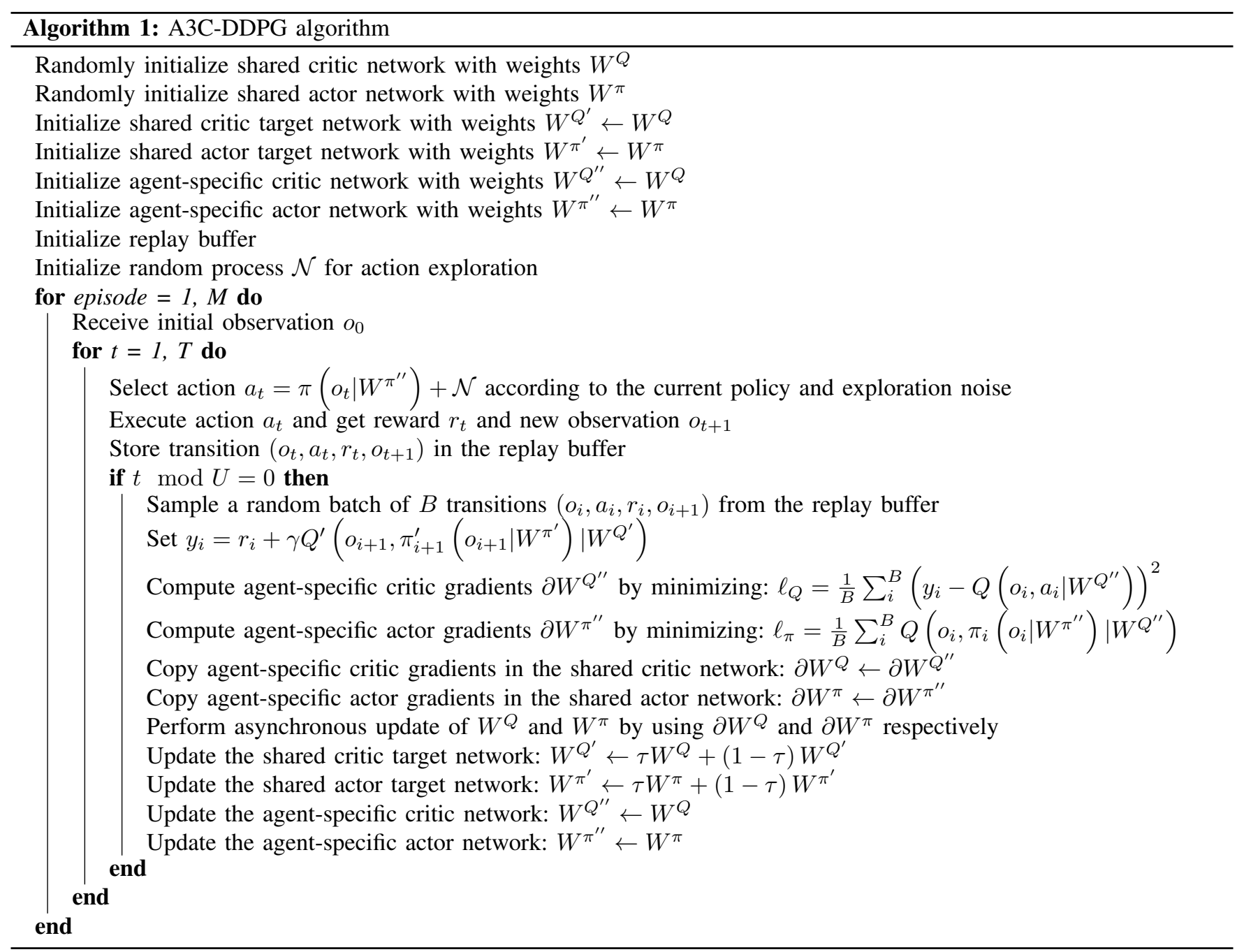

$8 \times 8$ filters with stride 4 , and the second with $324 \times 4$ filters with stride 2 . Both layers are followed by a $\operatorname{ReLU}$ activation and a GroupNorm layer. The image features extracted are further elaborated by a fully connected layer followed by a Gated Recurrent Unit (GRU) [43] network, both with 256 hidden nodes and ReLU activations. We decide to implement a GRU over a Long Short-Term Memory (LSTM) [44] network since, in most applications, the difference in terms 
of performance is negligible [45], [46], however, the former has fewer parameters, translating in a reduced computational complexity. The GRU output is then fed to 2 fully connected networks, with 200 and 100 neurons each and ReLU activations. At this point, the A-DNN produces the policy vector $\pi=\left[\pi_{\rho}, \pi_{\theta}\right]$ and the estimated distance $\hat{\rho}$ and angle $\hat{\theta}$ (which are discussed in the following sections), using a last dense layer with 4 neurons and tanh activation.

Since the vector $\pi$ is deterministic, to allow exploration of the MDP, a gaussian exploration noise $\mathcal{N}=\left[\mathcal{N}_{\rho}, \mathcal{N}_{\theta}\right]$, with mean $\mu$ and variance $\sigma^{2}$, is finally added to produce $a_{\rho}=\pi_{\rho}+\mathcal{N}_{\rho}$ and $a_{\theta}=\pi_{\theta}+\mathcal{N}_{\theta}$, which represent the speed and the angular speed, respectively, chosen by the actor. It is important to specify, that such exploration noise is applied during training only, and that, in test phase, the action vector $a=\left[a_{\rho}, a_{\theta}\right]$ coincides with $\pi$.

The C-DNN structure mimics that one of the actor, except for the output of the GRU layer, which is concatenated with the vector $\pi$ (from the actor network), before feeding it to the fully connected layers. Finally, the scalar action-value $Q$ is produced by a last fully connected layer with linear activation.

2) DDPG Losses: To update the network weights, we apply the standard losses of the DDPG algorithm. Hence, for the critic we have:

$$
\ell_{Q}=\frac{1}{T} \sum_{t}^{T}\left(y_{t}-Q\left(o_{t}, a_{t}\right)\right)^{2},
$$

where

$$
y_{t}=r_{t}+\gamma Q^{\prime}\left(o_{t+1}, \pi_{t+1}^{\prime}\right)
$$

and for the actor:

$$
\ell_{\pi}=\frac{1}{T} \sum_{t}^{T} Q\left(o_{t}, \pi_{t}\right) .
$$

Since performing the bootstrapping for the next observation $o_{t+1}$ with the same learned networks could lead to divergence, we create a copy of the actor and critic networks for calculating the target values $\pi^{\prime}$ and $Q^{\prime}$, respectively (Eq. (7)). These targets are needed to stabilise learning, and their parameters $\left(w^{\prime}\right)$ are updated by slowly tracking the ones $(w)$ of the learned networks: $w^{\prime} \leftarrow \tau w+(1-\tau) w^{\prime}$, with $\tau \ll 1$. As already mentioned, the data are sampled from different replay buffers of size $n$, which serve the dual purpose of minimising correlations between samples and speed up learning. Further details on the DDPG algorithm can be found in the original paper [39].

3) Heuristic Trajectories: Since, at the beginning of the episodes, the target can be spawned outside of the field of view of the tracker, as explained in Section III-B, the reward observed by the agent can be considerably low and sparse. This is a common problem in RL and, especially for challenging applications like VAT, may entirely compromise the whole training process. To avoid that, we design an effective deterministic tracking algorithm that we refer to as Heuristic Trajectories Generator (HTG). Since it uses ground truth informations provided by the simulation engine, we

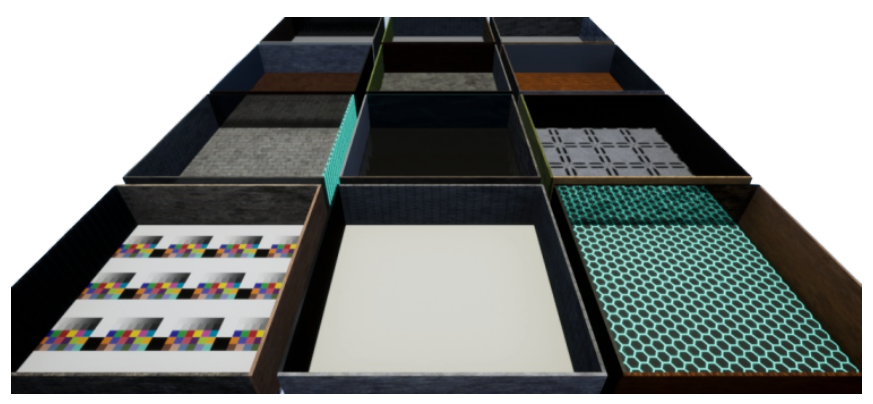

Fig. 4. An overview of the training environments. Since we employ parallel training, each tracker-target pair is placed inside one of the rooms. We employ a set of several different textures to randomize all the environments.

utilize it to fill the replay buffers with very useful trajectories just from the beginning of training. In particular, it employs the following heuristic:

$$
\pi_{\theta}^{h}=-\min \left(\frac{2\left|\theta_{t}-\theta^{*}\right|}{\mathrm{FOV}}, 1\right) \operatorname{sgn}\left(\theta_{t}\right),
$$

and

$$
\begin{aligned}
& \pi_{\rho}^{h}= \\
& \begin{cases}\min \left(\frac{\left|\rho_{t}-\rho^{*}\right|}{\rho_{\max }}, 1\right) \operatorname{sgn}\left(\rho_{t}-\rho^{*}\right), & \text { if }\left|\theta_{t}-\theta^{*}\right|<10 \\
0, & \text { otherwise }\end{cases}
\end{aligned}
$$

where FOV is the field of view of the tracker. During training, the actual action vector is then calculated by adding the gaussian exploration noise $\mathcal{N}$ to the vector $\pi^{h}=\left[\pi_{\rho}^{h}, \pi_{\theta}^{h}\right]$ : $a^{h}=\left[a_{\rho}^{h}, a_{\theta}^{h}\right]=\left[\pi_{\rho}^{h}+\mathcal{N}_{\rho}, \pi_{\theta}^{h}+\mathcal{N}_{\theta}\right]$. This is needed to guarantee that the heuristic trajectories contain enough variability to be valuable for training the critic. It should be noticed that, also because of the additive noise, the sequences of actions produced by this simple policy are not (and are not intended to be) optimal. However, as we show in Section [V] they demonstrate to be crucial for a successful training since remarkably useful for the critic network, which can start to evaluate reasonable policies right from the very beginning of training. It is also important to highlight that, contrary to Imitation Learning [47], [48], in which example trajectories are used to directly push the learned policy toward a predefined, and possibly suboptimal, behaviour, this technique simply augments the trajectories distribution that the critic observes.

4) Auxiliary Loss: To help the agent learn basic tracking concepts, we add an auxiliary task in which the actor network has to estimate the relative angle and distance with respect to the target. In particular, we introduce the following loss:

$$
\begin{aligned}
\ell_{a}=\frac{1}{L} \sum_{t}^{L}\left(\left(\hat{\rho}_{t}-\rho_{t}\right)^{2}+\left(\hat{\theta}_{t}-\theta_{t}\right)^{2}\right)+ \\
\quad+\frac{1}{L} \sum_{t}^{L}\left(\left(\hat{\rho}_{t}-\hat{\rho}_{t-1}\right)^{2}+\left(\hat{\theta}_{t}-\hat{\theta}_{t-1}\right)^{2}\right),
\end{aligned}
$$

where $L$ is the sequence length processed by the GRU, and $\hat{\rho}_{t}$ and $\hat{\theta}_{t}$ are, respectively, the distance and the angle estimated 


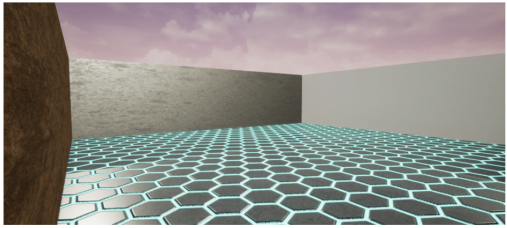

Training Environments
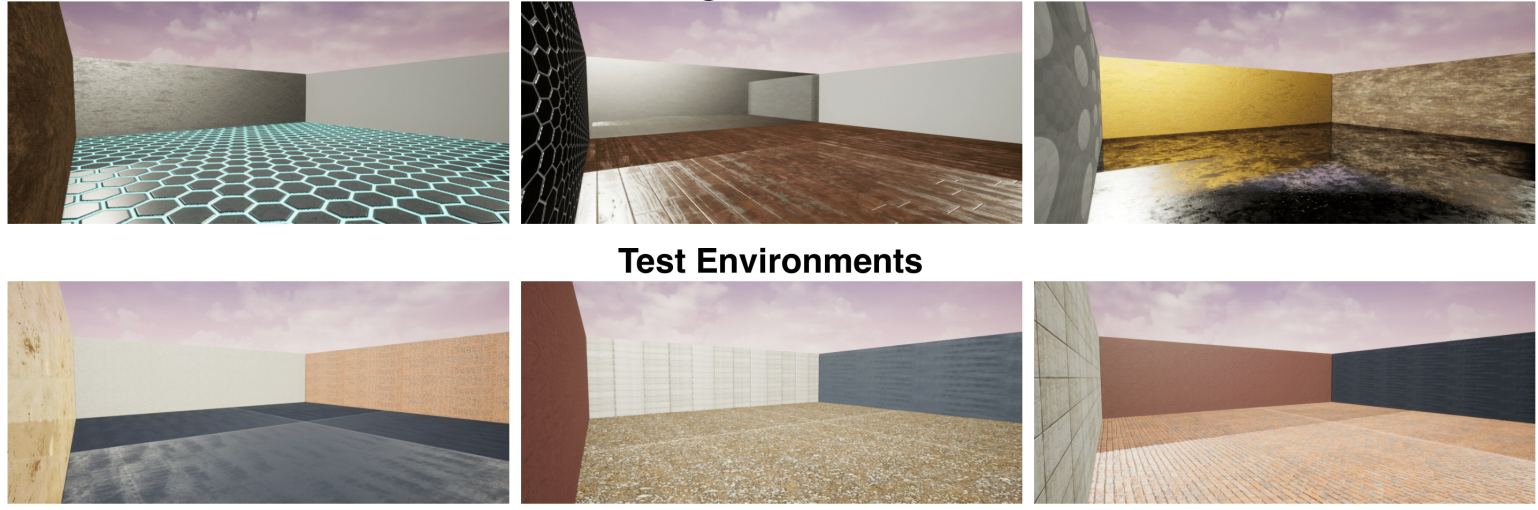

Fig. 5. Some examples of randomized textures used in our training (top row) and test (bottom row) environments. The textures used during the learning phase differ significantly from those used for the evaluation.

by the A-DNN at time $t$. The first term of the equation is the simple Mean Squared Error (MSE) between the estimated and the true values, while the second one is used to add temporal consistency to consecutive predictions.

To summarize, the overall optimization objective $\mathcal{L}$ for our C-VAT model can be written as:

$$
\mathcal{L}=\ell_{Q}+\ell_{\pi}+\ell_{a}
$$

\section{Environment}

To train our model, we build a simulated environment (see Fig. 4) using the photorealistic graphics engine Unreal Engine 4 (UE4) $)^{1}$. The environment consists of a large empty room, within which both the tracker and the target are positioned as follows: first, the tracker is randomly spawned in the room and, afterward, the target is randomly placed within a circumference of a predefined radius centered around the tracker.

Since our agent is trained in simulated environments only, in order to achieve generalization also to real world contexts, we apply domain randomization [23] to our synthetic scenario. This technique is successfully employed in many robotics and visual applications, such as: robotic harm control for object pushing [23], object detection [37] and robotic grasping [38]. It requires to randomize the training environment settings in order to make the system more robust to domain changes. Specifically, each time an episode ends we randomly change the lights conditions and the texture patterns of the rooms, including those of the walls, of the floor and of the target.

\section{EXPERIMENTS}

In the experiments, we aim to measure our C-VAT system performances, particularly w.r.t. environments not used for training. Specifically, we analyze our agents ability to: i) maintain the target to the desired relative angle $\theta^{*}$ and distance $\rho^{*}$, and ii) to recover tracking in case the target goes out-of-sight. To do that, we design several kind of tests, which are illustrated in the next sections.

\footnotetext{
${ }^{1}$ https://www.unrealengine.com
}

TABLE I

SETTINGS AND HYPERPARAMETERS

\begin{tabular}{cc}
\hline Hyperparameter & Value \\
\hline Reward coeff. $(A)$ & 0.1 \\
Optimal distance $\left(\rho^{*}\right)$ & $50 \mathrm{~cm}$ \\
Max distance from optimal $\left(\rho_{\max }\right)$ & $20 \mathrm{~cm}$ \\
Optimal angle $\left(\theta^{*}\right)$ & $0^{\circ}$ \\
Max angle from optimal $\left(\theta_{\max }\right)$ & $10^{\circ}$ \\
Exploration noise mean $(\mu)$ & 0 \\
Exploration noise std $(\sigma)$ & 0.5 \\
$\tau$ & 0.01 \\
Number of agents & 10 \\
Learning rate & 0.0001 \\
Batch size $(B)$ & 128 \\
Episode length & $250 \mathrm{steps}$ \\
Number of episodes $(M)$ & 9000 \\
HTG episode length & $70 \mathrm{steps}$ \\
Replay buffer size per agent $(n)$ & 3,500 trajectories \\
FOV & $90^{\circ}$ \\
Agent speed & {$[-8,8] \mathrm{cm} / \mathrm{s}$} \\
Agent steering speed & {$[-8,8] \mathrm{deg} / \mathrm{s}$} \\
Sequence length $(L)$ & $5 \mathrm{steps}$ \\
Network update interval $(U)$ & $25 \mathrm{steps}$ \\
Discount factor $(\gamma)$ & 0.99 \\
\hline
\end{tabular}

We compare and discuss the performance of C-VAT against two state-of-the-art baselines, the first one devised for discrete action spaces, while the second one for continuous control. We also propose an ablation study to evaluate the benefits brought by the HTG trajectories and the auxiliary angle-distance loss on the learning process and the final performance. Finally, to verify the generalization capability of our algorithm, we deploy the C-VAT model trained in the simulated environment (with no fine-tuning) in a robot within a real world scenario.

\section{A. Implementation Details}

We train both networks with $10 \mathrm{~A} 3 \mathrm{C}$ [41] agents for 9,000 episodes using the Adam optimizer, with initial learning rate 
TABLE II

EXPERIMENTAL RESULTS IN SIMULATED ENVIRONMENTS

\begin{tabular}{|c|c|c|c|c|c|c|c|c|}
\hline & & $\mathrm{HTG}+\mathcal{N}$ & HTG & $\begin{array}{c}\text { C-VAT } \\
\text { (No HT-AL) }\end{array}$ & $\begin{array}{c}\text { C-VAT } \\
\text { (No AL) }\end{array}$ & AOT [6] & $\mathrm{AD}-\mathrm{VAT}+[7]$ & C-VAT \\
\hline \multirow{4}{*}{ dynamic_target $(3,3)$} & $p_{\rho}$ & 0.90 & 0.95 & 0.14 & 0.99 & 0.76 & 0.83 & 0.99 \\
\hline & $p_{\theta}$ & 0.59 & 0.64 & 0.09 & 0.94 & 0.73 & 0.77 & 0.94 \\
\hline & $p_{c}$ & 0.75 & 0.80 & 0.12 & 0.97 & 0.74 & 0.80 & 0.96 \\
\hline & $p_{v}$ & 0.98 & 1.00 & 0.20 & 1.00 & 0.79 & 0.91 & 1.00 \\
\hline \multirow{4}{*}{ dynamic_target $(5,5)$} & $p_{\rho}$ & 0.72 & 0.94 & 0.15 & 0.98 & 0.55 & 0.86 & 0.98 \\
\hline & $p_{\theta}$ & 0.36 & 0.41 & 0.10 & 0.89 & 0.51 & 0.79 & 0.89 \\
\hline & $p_{c}$ & 0.54 & 0.67 & 0.12 & 0.94 & 0.53 & 0.83 & 0.94 \\
\hline & $p_{v}$ & 0.82 & 1.00 & 0.22 & 1.00 & 0.57 & 0.93 & 1.00 \\
\hline \multirow{4}{*}{ dynamic target $(8,8)$} & $p_{\rho}$ & 0.32 & 0.29 & 0.19 & 0.94 & 0.12 & 0.79 & 0.94 \\
\hline & $p_{\theta}$ & 0.19 & 0.06 & 0.14 & 0.51 & 0.10 & 0.50 & 0.65 \\
\hline & $p_{c}$ & 0.25 & 0.18 & 0.17 & 0.72 & 0.11 & 0.65 & 0.80 \\
\hline & $p_{v}$ & 0.39 & 0.31 & 0.30 & 0.99 & 0.14 & 0.85 & 0.99 \\
\hline \multirow{4}{*}{ dynamic_target $(8,3)$} & $p_{\rho}$ & 0.31 & 0.31 & 0.08 & 0.87 & 0.17 & 0.67 & 0.87 \\
\hline & $p_{\theta}$ & 0.21 & 0.21 & 0.05 & 0.87 & 0.15 & 0.65 & 0.87 \\
\hline & $p_{c}$ & 0.26 & 0.26 & 0.06 & 0.87 & 0.16 & 0.66 & 0.87 \\
\hline & $p_{v}$ & 0.85 & 0.66 & 0.15 & 0.95 & 0.20 & 0.77 & 0.94 \\
\hline \multirow{4}{*}{ random_target } & $p_{\rho}$ & 0.84 & 0.93 & 0.05 & 0.91 & 0.85 & 0.74 & 0.98 \\
\hline & $p_{\theta}$ & 0.77 & 0.84 & 0.03 & 0.90 & 0.82 & 0.79 & 0.96 \\
\hline & $p_{c}$ & 0.80 & 0.88 & 0.04 & 0.91 & 0.83 & 0.77 & 0.97 \\
\hline & $p_{v}$ & 0.99 & 1.00 & 0.10 & 0.94 & 0.88 & 0.86 & 1.00 \\
\hline \multirow{4}{*}{ adversarial target } & $p_{\rho}$ & 0.52 & 0.55 & 0.06 & 0.78 & 0.08 & 0.51 & 0.80 \\
\hline & $p_{\theta}$ & 0.45 & 0.49 & 0.05 & 0.78 & 0.07 & 0.35 & 0.80 \\
\hline & $p_{c}$ & 0.49 & 0.52 & 0.06 & 0.78 & 0.07 & 0.43 & 0.80 \\
\hline & $p_{v}$ & 0.73 & 0.71 & 0.16 & 0.88 & 0.10 & 0.58 & 0.90 \\
\hline
\end{tabular}

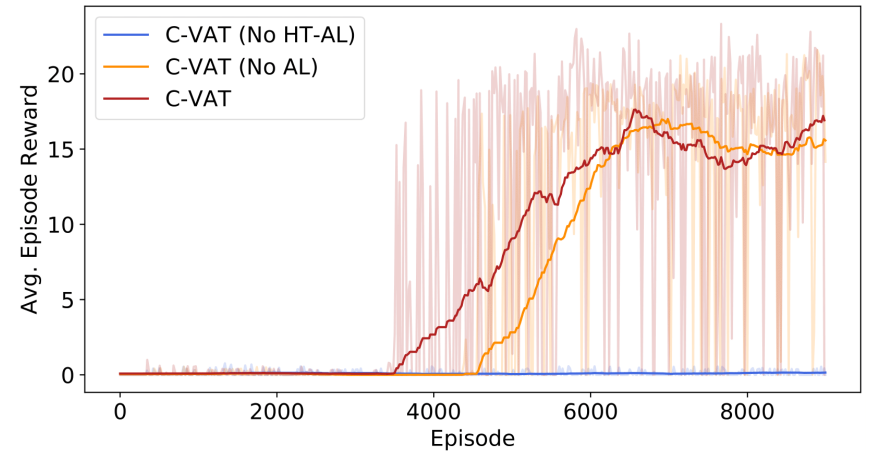

Fig. 6. Average episode reward during training. The figure shows that the heuristic trajectories are necessary to the development of an effective policy. C-VAT (No AL) and C-VAT approximately reach the same level of performance, however, the plot demonstrates that the auxiliary angledistance loss is helpful to speed up the learning process.

of 0.0001 and by setting the batch size to 128 . The episodes performed by the A-DNN are composed by 250 steps, while the episodes collected using the HTG have length 70. In order to compensate for the episode length difference, we insert the trajectories in the replay buffer with a ratio of $\frac{1}{4}$ between the trajectories of the actor and those generated by the HTG.

All the experiments are run by using a workstation equipped: $2 \times$ NVIDIA GTX 2080Ti with 11GB of VRAM, Intel Core processor i7-9800X $(3.80 \mathrm{GHz} \times 16)$ and $64 \mathrm{~GB}$ of DDR4 RAM. With this configuration, our model training takes about 140 minutes (roughly $1 s$ per episode), while, during the test phase, its forward pass requires $2 \mathrm{~ms}$ per frame on average.

The specific training and environment parameter settings that are used throughout our experiments are given in detail in Table II For a better understanding of the testing scenarios, several examples of simulated and real experiments can be examined in the video attachment.

\section{B. Evaluation Scenarios, Metrics and Models}

To demonstrate the validity of our approach, we run a large variety of simulated experiments in a new set of previously unknown environments (Fig. 5). We also compare our C-VAT method against 6 baselines: 
- HTG $+\mathcal{N}$ : the algorithm used during training to collect the heuristic trajectories;

- HTG: the algorithm used during training to collect the heuristic trajectories, without the exploration noise $\mathcal{N}$;

- C-VAT (No HT-AL): the basic version of our approach (A3C + DDPG);

- C-VAT (No AL): the model that is trained by using also the heuristic trajectories from the HTG but without the auxiliary distance-angle loss $\ell_{a}$ (Eq. (11));

- Active Object Tracking (AOT): the state-of-the-art approach proposed in [6]. We consider the continuous action space variant, since it is more similar to our approach than its discrete counterpart. As in the original paper, the model is trained against a target that follows randomly generated trajectories, which we refer to as random_target;

- AD-VAT+: the state-of-the-art approach proposed in [7]. As in the original work, the model policy is learned during the adversarial duelling training.

C-VAT (No HT-AL), C-VAT (No AL) and C-VAT are all tested without the exploration noise $\mathcal{N}$, and, as AOT, can move at a speed and steering speed within the range $[-8,8]$ $\mathrm{cm} / \mathrm{s}$ and $[-8,8] \mathrm{deg} / \mathrm{s}$, respectively. Conversely, AD-VAT+ can only perform the discrete actions, with speed $8 \mathrm{~cm} / \mathrm{s}$ and steering speed $8 \mathrm{deg} / \mathrm{s}$, in the set: \{move-forward, movebackward, turn-left, turn-right, turn-left-and-move-forward, turn-right-and-move-forward, no-op $\}$, as explained in [7].

To evaluate the agents performances, we design 3 different kinds of simulated experiments:

- dynamic_target: the target is spawned in front of the tracker and performs a circular trajectory in alternating random directions;

- random_target: the target is spawned in front of the tracker and performs randomly generated trajectories;

- adversarial_target: the target is spawned in front of the tracker and follows the policy learned during the adversarial duelling training [7];

and 4 metrics:

$$
\begin{gathered}
p_{\rho_{t}}=\left\{\begin{array}{ll}
\max \left(0,1-\frac{\left|\rho_{t}-\rho^{*}\right|}{150}\right), & \text { if }\left|\theta_{t}-\theta^{*}\right|>\frac{\mathrm{FOV}}{2} \\
0, & \text { otherwise }
\end{array},\right. \\
p_{\theta_{t}}= \begin{cases}\max \left(0,1-\frac{2\left|\theta_{t}-\theta^{*}\right|}{\mathrm{FOV}}\right), & \text { if }\left|\rho_{t}-\rho^{*}\right|>150 \\
0, & \text { otherwise }\end{cases} \\
p_{c_{t}}=\frac{p_{\rho_{t}}+p_{\theta_{t}}}{2}, \\
p_{v_{t}}= \begin{cases}1, & \text { if } p_{c_{t}}>0 \\
0, & \text { otherwise }\end{cases}
\end{gathered}
$$

The first $\left(p_{\rho_{t}}\right)$ and second $\left(p_{\theta_{t}}\right)$ metrics measure the tracker ability to maintain the specified distance and angle, respectively, from the target. It should be noted that both scores are 0 if the target exceeds the allowed distance $(150 \mathrm{~cm})$ or angle $\left(\frac{\mathrm{FOV}}{2}\right) \cdot p_{c_{t}}$ is simply the average of the first two metrics, and $p_{v_{t}}$ represents the percentage of steps in which the tracker has the target in view. All these metrics are averaged over the number of steps per run and by the number of runs.

All the experiments have the same fixed length duration of 250 steps. In order to evaluate the models performance for different target velocities, in the dynamic_target scenario, we choose to vary its speed and steering speed within the set $\{(3,3),(5,5),(8,8),(8,3)\}$. Since the adversarial_target experiments employs a target trained with a fixed speed and steering speed (equals to $8 \mathrm{~cm} / \mathrm{s}$ and $8 \mathrm{deg} / \mathrm{s}$, respectively), we decide to maintain the same values also for testing. All the aforementioned scenarios are averaged over 20 runs.

\section{Results - Synthetic Experiments}

The results of the simulated experiments are summarized in Table III. As can be observed, our approach is the one that shows the best overall performances. In particular, it behaves very similarly to C-VAT (No AL), which, however, in some scenarios (see dynamic_target $(8,8)$, random_target and adversarial_target), appears to be less accurate, as reflected by both $p_{\rho}$ and $p_{\theta}$ metrics. From the poor results of C-VAT (No HT-AL), it is evident that training with heuristic trajectories has contributed significantly to the development of our tracker capabilities. This is also confirmed by the curves in Fig. 6, which represent our models' average episode reward throughout training.

Both C-VAT (No AL) and C-VAT outperform AD-VAT+ in all scenarios and metrics. This may be due to the continuous action space they features, which allows them to track the target more accurately. Interestingly, the larger gap is observed with the adversarial_target, which proves capable to systematically elude AD-VAT+. By qualitatively analyzing the experiments (see the video attachment), the adversarial_target seems to have developed a clever policy that exploits the fact that AD-VAT+ cannot simultaneously move backward and turning. This strategy, however, is much less effective against C-VAT, which benefits from a symmetrical action space.

Our approach shows much better performance also with respect to AOT, especially against the targets with higher speeds (dynamic_target $(8,8)$, dynamic_target $(8,3)$ and adversarial_target). This is caused by the fact that during training AOT does not learn to lower its entropy in action selection and consequently the tracking policy appears quite "noisy". Because of that, it becomes rather common for the tracker to lose its target when it moves very quickly.

Remarkably, C-VAT dramatically outperforms also the HTG, both the noisy and non-noisy variants. As already explained in Section III-C.3 the heuristic trajectories are only needed in the early training stage, where the reward is still sparse. Indeed, as the results demonstrate, the C-VAT learned policy is not limited by the HTG trajectories.

\section{Results - Real Experiments}

With these experiments, we want to asses C-VAT generalization capabilities and its robustness in real conditions. To this aim, we deploy our algorithm, without any kind 

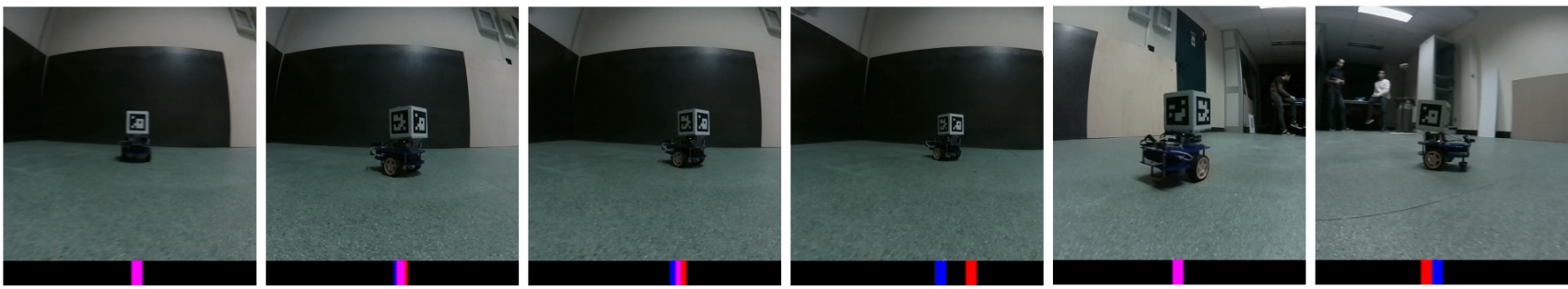

Fig. 7. Six images captured by the camera mounted on the tracker robot during the real world experiments. At the bottom of each figure, the blue and the red bars represent the current angle estimated by C-VAT and the one computed by the Aruco detector [49], respectively. As it can be observed, the two estimates are very similar and often coincide. The fact that, in some cases, the difference between them increases can be explained by noticing that the Aruco markers are not placed at the center of the target. It is important to highlight that C-VAT does not use any sort of marker to perform tracking (neither for training nor for testing). They are only used by the Aruco detector in order to provide a quantitative evaluation of our approach performance.
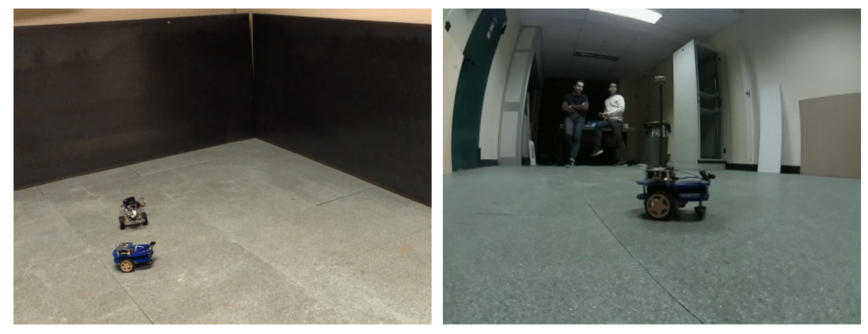

Fig. 8. Two images captured from the recording camera (left) and the tracker robot point of view (right) during the real world experiments. The visual appearance of our indoor environment is extremely different from that one of the simulated scenarios used during training.

TABLE III

EXPERIMENTAL RESULTS IN REAL ENVIRONMENTS

\begin{tabular}{c|cccc}
\hline Run & $\boldsymbol{p}_{\boldsymbol{\rho}}$ & $\boldsymbol{p}_{\boldsymbol{\theta}}$ & $\boldsymbol{p}_{\boldsymbol{c}}$ & $\boldsymbol{p}_{\boldsymbol{v}}$ \\
\hline 1 & 0.72 & 0.85 & 0.78 & 0.89 \\
2 & 0.74 & 0.83 & 0.79 & 0.89 \\
\hline
\end{tabular}

of fine-tuning, in an indoor environment on a real twowheeled mobile robot (Fig. 7, 8). Both the tracker and the target robots can perform the same actions as their simulated counterparts. The former is controlled by our CVAT model residing in a remote host, while the latter is manually controlled. Since, in the real world, we do not have access to ground truth informations, we equip the target robot with Aruco markers and use the Aruco detector algorithm [49] to approximate the real relative angle and distance from the tracker. In frames where measurements could not be obtained, we decide to calculate them by linear interpolation with those of adjacent frames. It is important to specify that the markers are used only for evaluation, since our method does not need them to perform tracking.

All the numerical results are reported in Table III Although C-VAT exhibits lower performance than those in simulation, it is still able to achieve remarkable results also in a real world environment. It should be noticed that this test scenario significantly differs from the simulated ones in terms of visual appearance. In particular, it is characterized by various objects, people, textures and lightning conditions that are completely absent in the training environments. Despite that, in Fig. 7) which shows the model angle estimation performances, it is shown that the robot is actually able to recognize and locate the target.

Additional qualitative results of these experiments are available in the attached video.

\section{CONCLUSIONS}

In this work, we presented a new DRL-based approach for Visual Active Tracking that deals with continuous action spaces. Through extensive experimentation, we showed that our approach can perform robust tracking in both synthetic and real environments. Despite the relevant complexity of training a continuous control policy for VAT, our novel learning procedure demonstrated to be able to produce an end-to-end tracking model more accurate and reliable than other state-of-the-art systems.

Although the results are promising, there are still many open problems and aspects to improve. Specifically, we intend to continue our work by addressing more complicated scenarios, such as multi-target tracking, which introduces new challenges and requires more sophisticated methods.

\section{REFERENCES}

[1] H. Nam and B. Han, "Learning multi-domain convolutional neural networks for visual tracking," in Proceedings of the IEEE conference on computer vision and pattern recognition, 2016, pp. 4293-4302.

[2] Y. Song, C. Ma, L. Gong, J. Zhang, R. W. Lau, and M.-H. Yang, "Crest: Convolutional residual learning for visual tracking," in Proceedings of the IEEE International Conference on Computer Vision, 2017, pp. 2555-2564.

[3] D. Zhang, H. Maei, X. Wang, and Y.-F. Wang, "Deep reinforcement learning for visual object tracking in videos," arXiv preprint arXiv:1701.08936, 2017.

[4] S. Pu, Y. Song, C. Ma, H. Zhang, and M.-H. Yang, "Deep attentive tracking via reciprocative learning," in Advances in Neural Information Processing Systems, 2018, pp. 1931-1941

[5] Y. Çelik, M. Altun, and M. Güneş, "Color based moving object tracking with an active camera using motion information," in 2017 International Artificial Intelligence and Data Processing Symposium (IDAP). IEEE, 2017, pp. 1-4.

[6] W. Luo, P. Sun, F. Zhong, W. Liu, T. Zhang, and Y. Wang, "End-to-end active object tracking and its real-world deployment via reinforcement learning," IEEE transactions on pattern analysis and machine intelligence, 2019.

[7] F. Zhong, P. Sun, W. Luo, T. Yan, and Y. Wang, "Ad-vat+: An asymmetric dueling mechanism for learning and understanding visual active tracking," IEEE transactions on pattern analysis and machine intelligence, 2019.

[8] J. Redmon, S. Divvala, R. Girshick, and A. Farhadi, "You only look once: Unified, real-time object detection," in Proceedings of the IEEE conference on computer vision and pattern recognition, 2016, pp. 779788 . 
[9] R. Mur-Artal and J. D. Tardós, "Orb-slam2: An open-source slam system for monocular, stereo, and rgb-d cameras," IEEE Transactions on Robotics, vol. 33, no. 5, pp. 1255-1262, 2017.

[10] M. Mancini, G. Costante, P. Valigi, and T. A. Ciarfuglia, "J-mod 2: Joint monocular obstacle detection and depth estimation," IEEE Robotics and Automation Letters, vol. 3, no. 3, pp. 1490-1497, 2018.

[11] J. Engel, V. Koltun, and D. Cremers, "Direct sparse odometry," IEEE transactions on pattern analysis and machine intelligence, vol. 40, no. 3, pp. 611-625, 2017.

[12] G. Costante and M. Mancini, "Uncertainty estimation for data-driven visual odometry," IEEE Transactions on Robotics, 2020

[13] S. Karaman and E. Frazzoli, "Sampling-based algorithms for optimal motion planning," The international journal of robotics research, vol. 30, no. 7, pp. 846-894, 2011.

[14] D. Murray and A. Basu, "Motion tracking with an active camera," IEEE transactions on pattern analysis and machine intelligence, vol. 16, no. 5, pp. 449-459, 1994.

[15] K. K. Kim, S. H. Cho, H. J. Kim, and J. Y. Lee, "Detecting and tracking moving object using an active camera," in The 7th International Conference on Advanced Communication Technology, 2005, ICACT 2005., vol. 2. IEEE, 2005, pp. 817-820.

[16] D. K. Das, M. Laha, S. Majumder, and D. Ray, "Stable and consistent object tracking: An active vision approach," in Advanced Computational and Communication Paradigms. Springer, 2018, pp. 299-308.

[17] L. Espeholt, H. Soyer, R. Munos, K. Simonyan, V. Mnih, T. Ward, Y. Doron, V. Firoiu, T. Harley, I. Dunning, et al., "Impala: Scalable distributed deep-rl with importance weighted actor-learner architectures," arXiv preprint arXiv:1802.01561, 2018.

[18] O. M. Andrychowicz, B. Baker, M. Chociej, R. Jozefowicz, B. McGrew, J. Pachocki, A. Petron, M. Plappert, G. Powell, A. Ray, et al., "Learning dexterous in-hand manipulation," The International Journal of Robotics Research, vol. 39, no. 1, pp. 3-20, 2020.

[19] Y. Zhu, R. Mottaghi, E. Kolve, J. J. Lim, A. Gupta, L. Fei-Fei, and A. Farhadi, "Target-driven visual navigation in indoor scenes using deep reinforcement learning," in 2017 IEEE international conference on robotics and automation (ICRA). IEEE, 2017, pp. 3357-3364.

[20] A. Devo, G. Costante, and P. Valigi, "Deep reinforcement learning for instruction following visual navigation in 3d maze-like environments," IEEE Robotics and Automation Letters, vol. 5, no. 2, pp. 1175-1182, 2020.

[21] A. Devo, G. Mezzetti, G. Costante, M. L. Fravolini, and P. Valigi, "Towards generalization in target-driven visual navigation by using deep reinforcement learning," IEEE Transactions on Robotics, 2020.

[22] J. Tobin, R. Fong, A. Ray, J. Schneider, W. Zaremba, and P. Abbeel, "Domain randomization for transferring deep neural networks from simulation to the real world," in 2017 IEEE/RSJ international conference on intelligent robots and systems (IROS). IEEE, 2017, pp. 23-30.

[23] X. B. Peng, M. Andrychowicz, W. Zaremba, and P. Abbeel, "Sim-toreal transfer of robotic control with dynamics randomization," in 2018 IEEE international conference on robotics and automation (ICRA). IEEE, 2018, pp. 1-8.

[24] Z. Zhu, Q. Wang, B. Li, W. Wu, J. Yan, and W. Hu, "Distractoraware siamese networks for visual object tracking," in Proceedings of the European Conference on Computer Vision (ECCV), 2018, pp. 101-117.

[25] Y. Song, C. Ma, X. Wu, L. Gong, L. Bao, W. Zuo, C. Shen, R. W. Lau, and M.-H. Yang, "Vital: Visual tracking via adversarial learning," in Proceedings of the IEEE Conference on Computer Vision and Pattern Recognition, 2018, pp. 8990-8999.

[26] S. Yun, J. Choi, Y. Yoo, K. Yun, and J. Young Choi, "Action-decision networks for visual tracking with deep reinforcement learning," in Proceedings of the IEEE conference on computer vision and pattern recognition, 2017, pp. 2711-2720.

[27] S. Ribaric, G. Adrinek, and S. Segvic, "Real-time active visual tracking system," in Proceedings of the 12th IEEE Mediterranean Electrotechnical Conference (IEEE Cat. No. 04CH37521), vol. 1. IEEE, 2004, pp. 231-234.

[28] N. Bellotto, B. Benfold, H. Harland, H.-H. Nagel, N. Pirlo, I. Reid, E. Sommerlade, and C. Zhao, "Cognitive visual tracking and camera control," Computer Vision and Image Understanding, vol. 116, no. 3, pp. 457-471, 2012.

[29] Z.-W. Hong, C. Yu-Ming, S.-Y. Su, T.-Y. Shann, Y.-H. Chang, H.K. Yang, B. H.-L. Ho, C.-C. Tu, Y.-C. Chang, T.-C. Hsiao, et al.,
"Virtual-to-real: Learning to control in visual semantic segmentation," arXiv preprint arXiv:1802.00285, 2018.

[30] M. Jaderberg, V. Mnih, W. M. Czarnecki, T. Schaul, J. Z. Leibo, D. Silver, and K. Kavukcuoglu, "Reinforcement learning with unsupervised auxiliary tasks," arXiv preprint arXiv:1611.05397, 2016.

[31] J. Kober, J. A. Bagnell, and J. Peters, "Reinforcement learning in robotics: A survey," The International Journal of Robotics Research, vol. 32, no. 11, pp. 1238-1274, 2013.

[32] T. G. Thuruthel, E. Falotico, F. Renda, and C. Laschi, "Model-based reinforcement learning for closed-loop dynamic control of soft robotic manipulators," IEEE Transactions on Robotics, vol. 35, no. 1, pp. 124 134, 2018.

[33] P. Mirowski, R. Pascanu, F. Viola, H. Soyer, A. J. Ballard, A. Banino, M. Denil, R. Goroshin, L. Sifre, K. Kavukcuoglu, et al., "Learning to navigate in complex environments," arXiv preprint arXiv:1611.03673, 2016

[34] S. Levine, C. Finn, T. Darrell, and P. Abbeel, "End-to-end training of deep visuomotor policies," The Journal of Machine Learning Research, vol. 17, no. 1, pp. 1334-1373, 2016.

[35] F. Sadeghi and S. Levine, "Cad2rl: Real single-image flight without a single real image," arXiv preprint arXiv:1611.04201, 2016.

[36] J. Li, J. Xu, F. Zhong, X. Kong, Y. Qiao, and Y. Wang, "Pose-assisted multi-camera collaboration for active object tracking," in Proceedings of the AAAI Conference on Artificial Intelligence, vol. 34, no. 01, 2020, pp. 759-766.

[37] J. Tremblay, A. Prakash, D. Acuna, M. Brophy, V. Jampani, C. Anil, T. To, E. Cameracci, S. Boochoon, and S. Birchfield, "Training deep networks with synthetic data: Bridging the reality gap by domain randomization," in Proceedings of the IEEE Conference on Computer Vision and Pattern Recognition Workshops, 2018, pp. 969-977.

[38] K. Bousmalis, A. Irpan, P. Wohlhart, Y. Bai, M. Kelcey, M. Kalakrishnan, L. Downs, J. Ibarz, P. Pastor, K. Konolige, et al., "Using simulation and domain adaptation to improve efficiency of deep robotic grasping," in 2018 IEEE International Conference on Robotics and Automation (ICRA). IEEE, 2018, pp. 4243-4250.

[39] T. P. Lillicrap, J. J. Hunt, A. Pritzel, N. Heess, T. Erez, Y. Tassa, D. Silver, and D. Wierstra, "Continuous control with deep reinforcement learning," arXiv preprint arXiv:1509.02971, 2015.

[40] R. S. Sutton and A. G. Barto, Reinforcement learning: An introduction. MIT press, 2018.

[41] V. Mnih, A. P. Badia, M. Mirza, A. Graves, T. Lillicrap, T. Harley, D. Silver, and K. Kavukcuoglu, "Asynchronous methods for deep reinforcement learning," in International conference on machine learning, 2016, pp. 1928-1937.

[42] Z. Wang, V. Bapst, N. Heess, V. Mnih, R. Munos, K. Kavukcuoglu, and N. de Freitas, "Sample efficient actor-critic with experience replay," arXiv preprint arXiv:1611.01224, 2016

[43] K. Cho, B. van Merriënboer, D. Bahdanau, and Y. Bengio, "On the properties of neural machine translation: Encoder-decoder approaches," Syntax, Semantics and Structure in Statistical Translation, p. 103, 2014.

[44] S. Hochreiter and J. Schmidhuber, "Long short-term memory," Neural computation, vol. 9, no. 8, pp. 1735-1780, 1997.

[45] J. Chung, C. Gulcehre, K. Cho, and Y. Bengio, "Empirical evaluation of gated recurrent neural networks on sequence modeling," arXiv preprint arXiv:1412.3555, 2014.

[46] S. Cascianelli, G. Costante, T. A. Ciarfuglia, P. Valigi, and M. L. Fravolini, "Full-gru natural language video description for service robotics applications," IEEE Robotics and Automation Letters, vol. 3 , no. 2, pp. 841-848, 2018.

[47] A. Billard, S. Calinon, R. Dillmann, and S. Schaal, "Survey: Robot programming by demonstration," Handbook of robotics, vol. 59, no. BOOK_CHAP, 2008.

[48] B. D. Argall, S. Chernova, M. Veloso, and B. Browning, "A survey of robot learning from demonstration," Robotics and autonomous systems, vol. 57, no. 5, pp. 469-483, 2009.

[49] S. Garrido-Jurado, R. Muñoz-Salinas, F. J. Madrid-Cuevas, and M. J. Marín-Jiménez, "Automatic generation and detection of highly reliable fiducial markers under occlusion," Pattern Recognition, vol. 47, no. 6 , pp. 2280-2292, 2014. 